\title{
The DNA-binding domain of CaNdt80p is required to activate CDR1 involved in drug resistance in Candida albicans
}

Correspondence

Hsiu-Jung Lo

hjlo@nhri.org.tw

Received 28 March 2006

Accepted 13 July 2006

\author{
Jang-Shiun Wang, ${ }^{1,2}$ Yun-Liang Yang, ${ }^{3}$ Chin-Jung Wu, ${ }^{3}$ Karen J. Ouyang, ${ }^{3}$ \\ Kuo-Yun Tseng, ${ }^{2}$ Chia-Geun Chen, ${ }^{2} \mathrm{Hsin} \mathrm{Wang}^{3}$ and Hsiu-Jung Lo ${ }^{2}$ \\ ${ }^{1}$ Graduate Institute of Life Sciences, National Defence Medical Center, Taipei, Taiwan, Republic \\ of China \\ ${ }^{2}$ Division of Clinical Research, National Health Research Institutes, 35 Keyan Road, Zhunan \\ Town, Miaoli County 350, Taiwan, Republic of China \\ ${ }^{3}$ Department of Biological Science and Technology, National Chiao Tung University, Hsinchu, \\ Taiwan, Republic of China
}

CaNdt80p, the Candida albicans homologue of the Saccharomyces cerevisiae transcription factor ScNdt80p, has been identified as a positive regulator of CDR1, which encodes an efflux pump involved in drug resistance in $C$. albicans. To investigate the involvement of the putative DNA-binding domain of CaNdt80p in drug resistance, chimeras of CaNdt80p and ScNdt80p were constructed. Interestingly, the DNA-binding domain of ScNdt80p could functionally complement that of CaNdt80p to activate CDR1p-lacZ in S. cerevisiae. Consistently, CaNdt80p containing a mutation in the DNA-binding domain failed to activate $C D R 1 p-l a c Z$ in $S$. cerevisiae. Furthermore, a copy of CaNDT80 with the same mutation also failed to complement the drug-sensitive phenotype caused by a null mutation in C. albicans. Thus, the DNA-binding domain of CaNdt80p is critical for its function in drug resistance in C. albicans.

\section{INTRODUCTION}

Candida albicans is the most frequently isolated fungal pathogen in humans, and has caused morbidity in seriously debilitated and immunocompromised hosts (Edwards, 1990). The prevalence of nosocomial candidaemia increased 27-fold from 1981 to 1993 at a hospital in Taiwan (Chen et al., 1997; Hung et al., 1996). In the USA, yeast infections also rank as the fourth-most-common cause of nosocomial bloodstream infection (Beck-Sague \& Jarvis, 1993; Pfaller et al., 1998). Coinciding with the increased usage of antifungal drugs, incidences of drug resistance have also multiplied (Pfaller et al., 2000; Vanden Bossche et al., 1994; Yang \& Lo, 2001).

Overexpression of efflux pumps, such as ATP-binding cassette $(\mathrm{ABC})$ transporters, has been shown to be the major mechanism for drug resistance in clinical isolates (Karababa et al., 2004; Marger \& Saier, 1993; Michaelis \& Berkower, 1995). Mutations on CDR1 in C. albicans result in an increased susceptibility to azole drugs (Sanglard et al., 1996), which is consistent with the observation that overexpression of CDR1 contributes to the drug resistance in clinical isolates (Lopez-Ribot et al., 1998). Recently, two transcription factors, CaNdt80p and Taclp, have been identified as

Abbreviation: $\beta$-Gal, $\beta$-galactosidase. positive regulators of $C D R 1$, and mutations of either gene result in an increased drug susceptibility (Chen et al., 2004; Coste et al., 2004).

CaNdt80p is a C. albicans homologue of the Saccharomyces cerevisiae ScNdt80p (Chen et al., 2004). Nevertheless, $\mathrm{CaNdt80} \mathrm{p}$ is reported to be involved in drug resistance in C. albicans (Chen et al., 2004), while ScNdt80p is a meiosisspecific transcription factor in S. cerevisiae (Abi-Said et al., 1997; Chu et al., 1998; Chu \& Herskowitz, 1998). CaNdt80p is 672 amino acids (aa) in length and ScNdt80p 592 aa (Fig. 1a). The sequence from amino acids 221 to 592 of CaNdt80p and that from amino acids 1 to 330 of ScNdt80p share $35 \%$ identity and $53 \%$ similarity (Fig. 1b). Interestingly, there is no similarity between the $\mathrm{N}$ terminal of CaNdt80p and the C terminal of ScNdt80p (Fig. 1a) (Chen et al., 2004). The amino acid residues from 1 to 330 of ScNdt80p have been shown to be important for the DNAbinding activity. The residues responsible for the DNAbinding capability of ScNdt80p have been reported. Among them, the 177Arg residue is the most important one for ScNdt80p to bind DNA. When this 177Arg residue is mutated, the DNA-binding capability decreases 27-fold (Montano et al., 2002). Whether the putative DNA-binding domain of CaNdt80p also plays an important role in its function(s) has not been reported. 


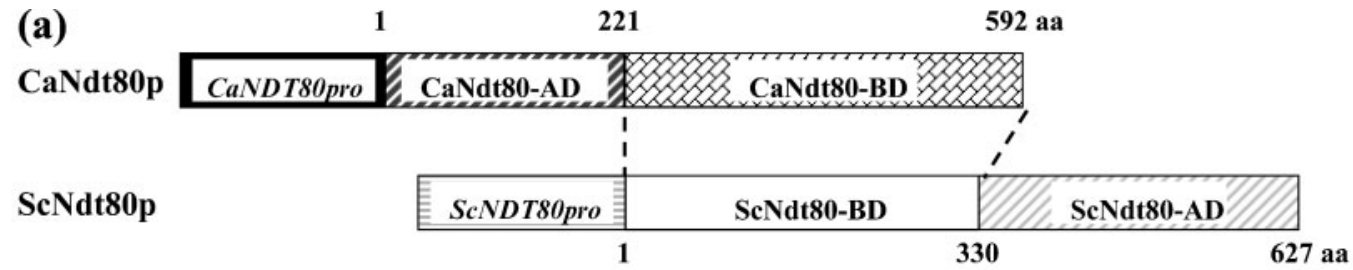

(b)
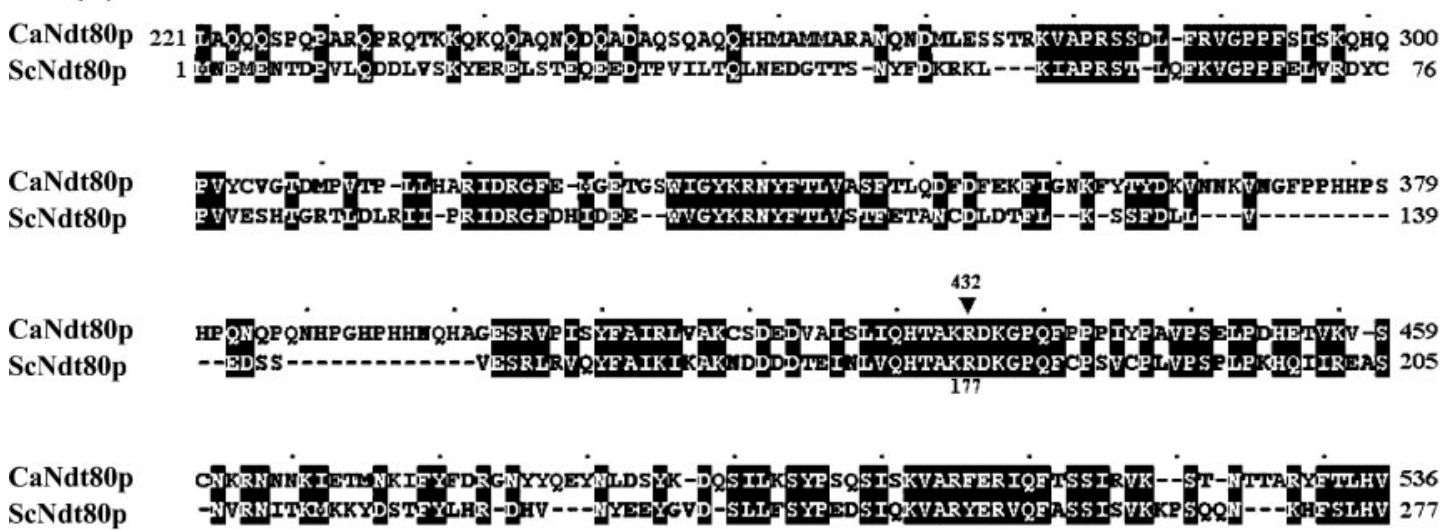

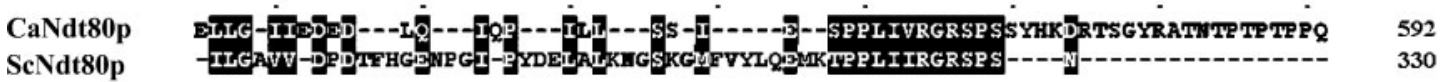

Fig. 1. Comparison of $\mathrm{CaNdt80p}$ in C. albicans and $\mathrm{ScNdt80p}$ in S. cerevisiae. (a) Overall comparison between CaNdt80p and ScNdt80p. For CaNdt80p, the promoter (pro) is in the solid black box, the activation domain (AD) is in the black diagonally hatched box, and the DNA-binding domain (BD) is in the cross-hatched box. For ScNdt80p, the promoter is in the horizontally lined box, the DNA-binding domain (BD) is in the open box, and the activation domain (AD) is in the grey diagonally hatched box. (b) Protein sequences of the DNA-binding domains of CaNdt80p and ScNdt80p. Similar residues of the two sequences are shaded. The mutated residue in $\mathrm{CaNdt80p}$ is indicated by an arrow, and the numbers represent the positions of amino acid residues in CaNdt80p and ScNdt80p.

In this study, we have constructed chimeras of CaNdt80p and ScNdt80p, and have found that the DNA-binding domain of ScNdt80p could functionally complement that of CaNdt80p to activate CDR1p-lacZ. Furthermore, complementation of a Candt80/Candt80 mutant with a copy of Candt80 carrying a mutation in the DNA-binding domain failed to restore drug resistance, in contrast to the wild-type CaNDT80 gene. In conclusion, the DNA-binding domain of CaNdt80p is critical for activating the CDR1 promoter and participating in the regulation of drug-resistance activity in C. albicans.

\section{METHODS}

Strains and media. S. cerevisiae strains, C. albicans strains, plasmids and primers used in this study are listed in Tables 1, 2, 3 and 4 , respectively. Yeast peptone dextrose (YPD; $1 \%$ yeast extract, $2 \%$ peptone, $2 \%$ glucose), and synthetic dextrose (SD; $0.67 \%$ yeast nitrogen base without amino acids, $2 \%$ glucose) were prepared as described in Sherman et al. (2002).

Domain swapping. The schematic diagram in Fig. 2 shows the various chimeras used in this study. The sequence containing the promoter and the $5^{\prime}$ portion of CaNDT80 (from 578 bp upstream to $666 \mathrm{bp}$ downstream of the translation initiation site; denoted as -578 to $666 \mathrm{bp}$ ) was amplified from LOB45 (Chen et al., 2004) by PCR with primers HJL382 and HJL383, HJL384 or HJL436, which introduced a SacII site at the $5^{\prime}$ end and a XmaI or XhoI site at the $3^{\prime}$ end. The DNA fragment containing the DNA-binding domain of ScNdt80p (from +4 to $+992 \mathrm{bp}$ ) was amplified from LOB46 (Chen et al., 2004) with the primers HJL385 and HJL387 to introduce a XmaI site at the $5^{\prime}$ end and a ClaI site at the $3^{\prime}$ end. The entire ORF of ScNDT80 (from +4 bp to $+2517 \mathrm{bp}$ ) was amplified by primers HJL385 and HJL386 to introduce a XmaI site at the $5^{\prime}$ end and a ClaI site at the $3^{\prime}$ end. The PCR products were cloned into PCRII-TOPO (Invitrogen, K65001) and sequenced. The inserted DNA fragments were then cloned into the high-copynumber vector pRS426 at appropriate restriction sites to construct plasmids LOB114 to LOB117. Different chimeras (Fig. 2) were used to transform S. cerevisiae strain SLO1 (Chen et al., 2004) containing the CDR1p-lacZ reporter to construct SLO75, -77, -79 and -81 . The LOB46 plasmid containing ScNDT80 was also used to transform the S. cerevisiae strain SLO1 (Chen et al., 2004), to construct SLO71.

In vitro assay of $\boldsymbol{\beta}$-galactosidase activity. The overnight $S$. cerevisiae cell cultures were diluted into $20 \mathrm{ml} \mathrm{SD}$ plus adenine and histidine (SD/Ade/His) medium in a $250 \mathrm{ml}$ flask to $\mathrm{OD}_{600} 0 \cdot 2$. The cells were harvested by centrifugation at 3000 r.p.m. for $10 \mathrm{~min}$ at $4{ }^{\circ} \mathrm{C}$ (Sorvall RT7) after being grown at $30^{\circ} \mathrm{C}$ for $4 \mathrm{~h}$. Each cell 
Table 1. S. cerevisiae strains used in this study

\begin{tabular}{|c|c|c|}
\hline Strain & Genotype & Source \\
\hline SLO1 & MATa his3:: hisG ura3-52 leu2:: hisG ade3:: CDR1p-lacZ-LEU2 & Chen et al. (2004) \\
\hline SLO5 & MATa his3:: hisG ura3-52 leu2:: hisG ade3:: CDR1p-lacZ-LEU2 (LOB45) & Chen et al. (2004) \\
\hline SLO71 & MATa his3:: hisG ura3-52 leu2:: hisG ade3:: CDR1p-lacZ-LEU2 (LOB46) & This study \\
\hline SLO79 & MATa his3:: hisG ura3-52 leu2:: hisG ade3:: CDR1p-lacZ-LEU2 (LOB116) & This study \\
\hline SLO81 & MATa his3:: hisG ura3-52 leu2:: hisG ade3:: CDR1p-lacZ-LEU2 (LOB117) & This study \\
\hline SLO85 & MATa his3:: hisG ura3-52 leu2:: hisG ade3:: CDR1p-lacZ-LEU2 (LOB119) & This study \\
\hline
\end{tabular}

Table 2. C. albicans strains used in this study

\begin{tabular}{|c|c|c|}
\hline Strain & Genotype & Source \\
\hline YLO132 & 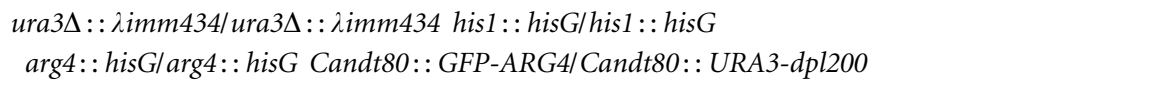 & Chen et al. (2004) \\
\hline YLO133 & 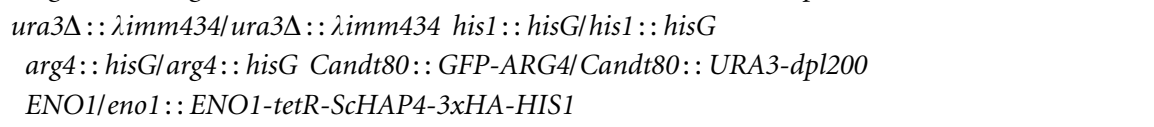 & Chen et al. (2004) \\
\hline YLO263 & 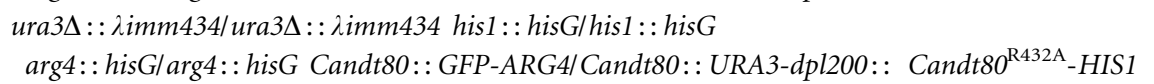 & This study \\
\hline
\end{tabular}

pellet was resuspended in $250 \mu \mathrm{l}$ breaking buffer ( $1 \mathrm{mM} \mathrm{DTT}, 20 \%$, v/v, glycerol, $0 \cdot 1 \mathrm{M}$ Tris/HCl, $\mathrm{pH} 8 \cdot 0$ ) with $12 \cdot 5 \mu \mathrm{l} 40 \mathrm{mM}$ PMSF, and placed on ice. Acid-washed glass beads were added to just below the liquid level, and the suspension was vortexed at maximum speed for $60 \mathrm{~s}$, followed by an additional incubation for $60 \mathrm{~s}$ at $4{ }^{\circ} \mathrm{C}$. The vortexing procedure was repeated four times, and then an extra $300 \mu \mathrm{l}$ breaking buffer was added. The cell extract was centrifuged at 3000 r.p.m. for $5 \mathrm{~min}$ at $4{ }^{\circ} \mathrm{C}$. The supernatant was transferred to a fresh ice-cold Eppendorf tube for $\beta$-galactosidase $(\beta$-Gal) assay and Bradford quantitative protein assay.
Yeast extract $(10 \mu \mathrm{l})$ was added to a $12 \times 75 \mathrm{~mm}$ disposable glass tube containing $990 \mu \mathrm{l}$ cold $\mathrm{Z}$ buffer $\left(60 \mathrm{mM} \mathrm{Na}_{2} \mathrm{HPO}_{4}, 40 \mathrm{mM} \mathrm{NaH} \mathrm{PO}_{4}\right.$, $\left.10 \mathrm{mM} \mathrm{KCl}, 1 \mathrm{mM} \mathrm{MgSO}_{4}\right)$ with $\beta$-mercaptoethanol $\left(2 \cdot 7 \mathrm{ml} \mathrm{l}^{-1}\right)$. A total of $200 \mu \mathrm{l}$ ONPG (Sigma, N1127; $4 \mathrm{mg} \mathrm{ml}^{-1}$ in $\mathrm{Z}$ buffer) as enzyme substrate was added to start the reaction at $t=0$. The reaction was stopped by adding $500 \mu \mathrm{l} 1 \mathrm{M} \mathrm{Na}_{2} \mathrm{CO}_{3}$. Bio-Rad Protein Assay Dye (Catalogue no. 500-0006) was used to determine the protein concentration of the cell extract. Based on the Bradford dye-binding procedure, the protein concentration of the supernatant was determined by comparison with a standard curve of $A_{595}$ versus

Table 3. Plasmids used in this study

\begin{tabular}{|c|c|c|}
\hline Plasmid & Description & Source \\
\hline pRS426 & - & Christianson et al. (1992) \\
\hline LOB45 & pRS426-CaNDT80 & Chen et al. (2004) \\
\hline LOB46 & pRS426-ScNDT80 & Chen et al. (2004) \\
\hline LOB49 & pGEM-HIS1-CaNDT80 & Chen et al. (2004) \\
\hline LOB114 & pRS426-CaNDT80p-CaNDT80 (AD)-ScNDT80 (BD) & This study \\
\hline LOB115 & pRS426-CaNDT80p-CaNDT80 (AD) & This study \\
\hline LOB116 & pRS426-CaNDT80p-ScNDT80 (ORF) & This study \\
\hline LOB117 & pRS426-CaNDT80p-CaNDT80 (AD)-ScNDT80 (ORF) & This study \\
\hline LOB119 & pRS426-Candt $80^{\mathrm{R} 432 \mathrm{~A}}$ & This study \\
\hline LOB122 & pGEM-HIS1-Candt $80^{\mathrm{R} 432 \mathrm{~A}}$ & This study \\
\hline
\end{tabular}


Table 4. Primers used in this study

\begin{tabular}{|c|c|c|}
\hline Name & Sequence & Location \\
\hline HJL237 & TTCССАССАССААТСТАСС & CaNDT80: +1311 to $+1330^{*}$ \\
\hline HJL238 & GTTTTGCTTACTGTGGAGGAG & CaNDT80: +1786 to +1766 \\
\hline HJL293 & AGTCATTGAACGAATTCTTGGCTG & CaEFB1: +38 to +61 \\
\hline HJL294 & TTCTTCAACAGCAGCTTGTAAGTC & CaEFB1: +591 to +568 \\
\hline HJL382 & CTTCCGCGGTTGTGGCGATTTTCACTTTC & CaNDT80: -578 to -559 \\
\hline HJL383 & TTTTCCCGGGCATCATTCTGACAAGAGTTT & CaNDT80: -14 to +6 \\
\hline HJL384 & ATTACCCGGGAGCCAAAGGTTGTGGTGTT & CaNDT80: +666 to +648 \\
\hline HJL385 & TAAACCCGGGAATGAAATGGAAAACACAGAT & ScNDT80: +4 to +24 \\
\hline HJL386 & GGATCTCGAGTGTCAAGCCAAATGCATAC & ScNDT80: +2517 to +2498 \\
\hline HJL387 & CCATCGATTATTAGTTAGAAGGTGATCTTC & ScNDT80: +992 to +974 \\
\hline HJL436 & GGATCTCGAGTTAAGCCAAAGGTTGTGGTGTT & CaNDT80: +666 to +648 \\
\hline HJL562 & GATCCAGCACACTGCTAAAGCAGATAAAGGTCCACAGTTCC & CaNDT80: +1275 to +1315 \\
\hline HJL567 & GGAACTGTGGACCTTTATCTGCTTTAGCAGTGTGCTGGATC & CaNDT80: +1315 to +1275 \\
\hline HJL800 & CCTAACGTATCGAGTGAAG & CaNDT80: +46 to +64 \\
\hline HJL801 & CATTGTAGTGCGACATCTG & CaNDT80: +337 to +319 \\
\hline HJL802 & CCAGAGATAGGCTCACG & ScNDT80: +1558 to +1574 \\
\hline HJL803 & CCAGATGGTAGAGTACCG & ScNDT80: +1832 to +1815 \\
\hline HJL804 & CATCCACCGATTTCTCCAAG & ScEFB1: +5 to +24 \\
\hline HJL805 & GTCTTCGTCTTCTTCAATGC & ScEFB1: +579 to +460 \\
\hline
\end{tabular}

${ }^{\star}$ The A of the ATG translation initiation site is +1 .

protein concentration, obtained by the serial twofold dilution of $500 \mu \mathrm{g} \mathrm{ml}^{-1}$ BSA down to $62 \cdot 5 \mu \mathrm{g} \mathrm{ml}^{-1}$. $\beta$-Gal activity units were calculated (moles ONPG cleaved per minute per milligram protein) by the following equation:

$\left(\mathrm{A}_{420} \times 378\right) /($ time $\times$ volume of extract $\times$ protein concentration $)$

where time is in minutes, volume of extract in millilitres, and protein concentration in milligrams per millilitre.

RT-PCR. We used two-step RT-PCR to detect the level of mRNA in different strains. Total RNA was isolated by the acid hot phenol method (Schmitt et al., 1990), followed by RQ1 RNase-free DNase (Promega, M610A) treatment to digest any contaminating DNA. First, $1 \mu \mathrm{g}$ total RNA from each strain was used as template with oligo-dT as primer to synthesize the first-strand cDNA with StrataScript Reverse Transcriptase (Strategene, 600085). The product of each reaction was diluted in sterile double-distilled $\mathrm{H}_{2} \mathrm{O}$, then used as the template to carry out the PCR amplification. Each reaction contained $50 \mu \mathrm{l}$ PCR mixture including $0.25 \mu \mathrm{M}$ each primer, $0.2 \mathrm{mM}$ dNTPs, $1 \times$ buffer $(10 \mathrm{mM}$ Tris/HCl, pH 9.0, $1.5 \mathrm{mM}$ $\mathrm{MgCl}_{2}, 50 \mathrm{mM} \mathrm{KCl}$ ) and $1 \mathrm{U} \mathrm{Taq}$ polymerase (Amersham, 27-079906). The reaction conditions were as follows: $95^{\circ} \mathrm{C}$ for $30 \mathrm{~s}, 57^{\circ} \mathrm{C}$ for $30 \mathrm{~s}$ and $72{ }^{\circ} \mathrm{C}$ for $80 \mathrm{~s}$ for 35 cycles. The primers HJL237 and HJL238 were used to detect the CaNDT80 mRNA in different $C$. albicans strains, and the primers HJL800 and HJL801 were used for CaNDT80 in different chimeras in S. cerevisiae strains. The primers HJL802 and HJL803 were used for the detection of ScNDT80. The primers HJL293 and HJL294, the sequences of which were deduced from the intron and exon of the C. albicans CaEFB1 gene, were used to distinguish the PCR products resulting from either the genomic DNA (928 bp DNA fragment) or the spliced RNA (564 bp DNA fragment) (Schaller et al., 2003). For S. cerevisiae, the primers HJL804 and HJL805 were used for the detection of ScEFB1.

Quantitative analysis of mRNA level by real-time PCR. The cells were harvested at $\mathrm{OD}_{600} 0 \cdot 7-0 \cdot 9$ after being grown in $20 \mathrm{ml} \mathrm{SD}$ liquid medium at $30^{\circ} \mathrm{C}$. Total RNA was isolated by the acid hot phenol method (Schmitt et al., 1990), followed by RQ1 RNase-free DNase (Promega, M610A) treatment to digest any contaminating DNA. A real-time PCR was performed in a Rotor-Gene 3000 instrument (Corbett Research) with a TITANIUM Taq PCR kit (BD Clontech, 639210) and SYBR Green I Nucleic Acid stain (Cambrex, 50513 ) to determine the level of mRNA. The sample set-up was carried out automatically by a CAS-1200 instrument (Corbett Research). The real-time PCR was performed according to the instructions of the manufacturer, and the expression of CaTEF3 in each strain was used as the loading control. The relative quantification was based on two standard curves for comparisons, and the results were given as a ratio (Kofron et al., 1999). The level of CaNDT80 mRNA isolated from the cells containing the wild-type CaNDT80 gene was assigned the value 1, and the relative level of mRNA isolated from different strains was normalized accordingly.

Site-directed mutagenesis. The Arg432 residue of CaNdt80p was altered by site-directed mutagenesis using the QuikChange SiteDirected Mutagenesis kit (Stratagene, 200519). The primers HJL562 and HJL567 were used to mutate the CaNdt80p 432Arg to Ala on LOB45 and LOB49, to construct LOB119 and LOB122, respectively. Then, LOB119 was used to transform the S. cerevisiae strain SLO1 (Chen et al., 2004) containing the CDR1p-lacZ reporter, to generate SLO85. LOB122 was linearized at the SpeI site located in the promoter of CaNDT80 before being used to transform the Candt80/ Candt80 homozygous mutant YLO132 (Chen et al., 2004) to generate the YLO263 (Candt80/Candt80::Candt80 ${ }^{\mathrm{R} 432 \mathrm{~A}}$ ) strain. All constructs were assessed by PCR and sequencing.

Antifungal susceptibility tests. The Etest (Pfaller et al., 2003; Tapia et al., 2003) was used to determine susceptibility to antifungal agents for C. albicans. Homogenized isolated colonies grown on SD medium overnight were transferred in $0.85 \% \mathrm{NaCl}$ to achieve a density of $5 \times 10^{6}$ cells $\mathrm{ml}^{-1}$. A sterile swab was dipped into inoculum suspension and then used to swab the entire agar surface of an SD plate evenly. Fluconazole Etest strips (AB BIODISK) were applied to 


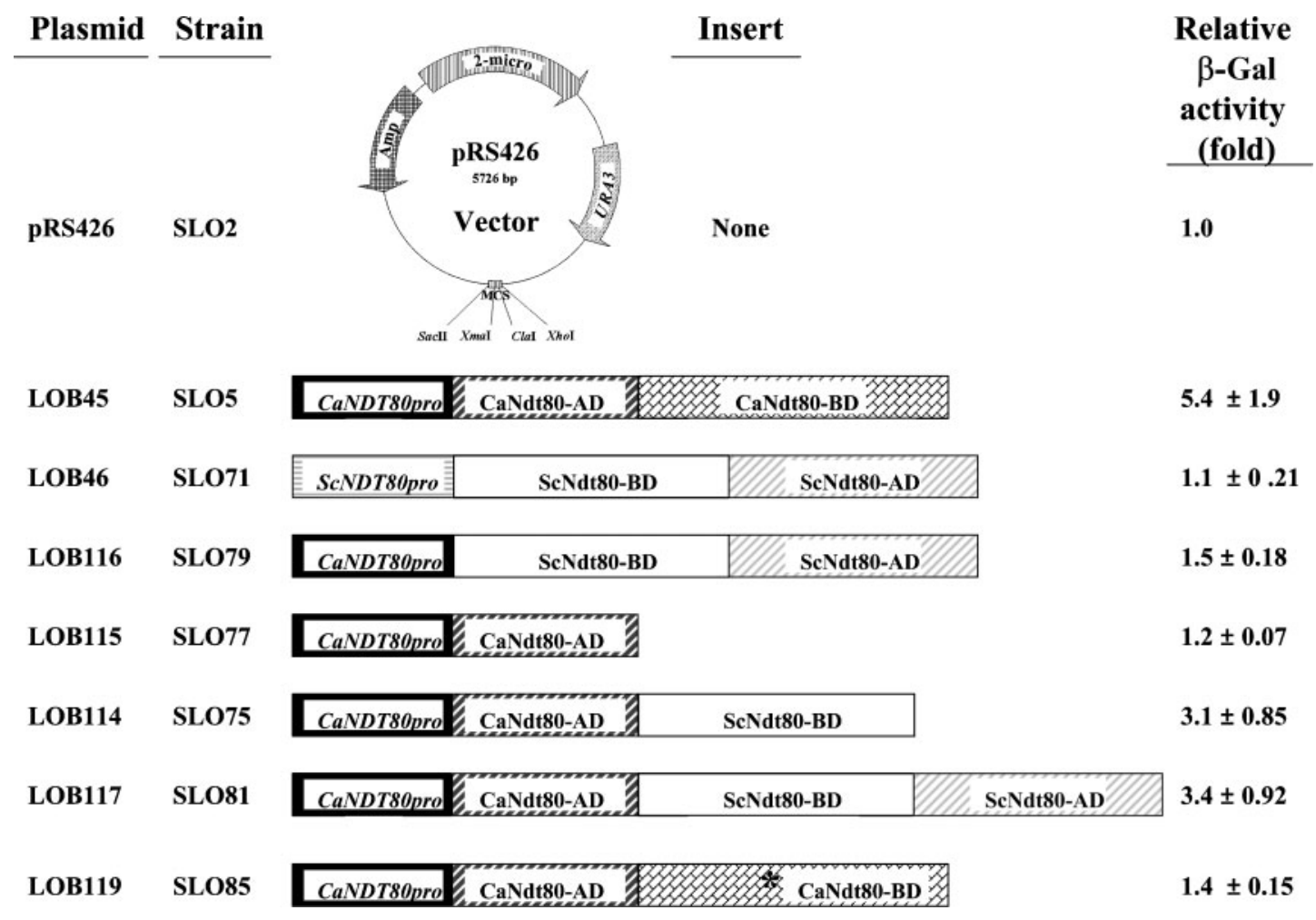

Fig. 2. Different chimeras having different effects on the expression of the CDR1p-lacZ reporter in $S$. cerevisiae. For CaNdt80p, the promoter (pro) is in the solid black box, the activation domain (AD) is in the black hatched box, and the DNAbinding domain (BD) is in the cross-hatched box. For ScNdt80p, the promoter is in the horizontally lined box, the DNA-binding domain (BD) is in the open box, and the activation domain (AD) is in the grey hatched box. The $\beta$-Gal activity of the strain (SLO2) containing vector alone (pRS426) was defined as 1 , and the $\beta$-Gal activity of cells containing different chimeras was normalized accordingly. The mutated residue is marked by an asterisk.

the plate when the excess moisture had been absorbed completely. The agar dilution method was also used to determine the susceptibility to antifungal agents of $C$. albicans. First, cells were diluted to $\mathrm{OD}_{600} 2\left(\sim 2 \times 10^{7}{\left.\text { cells } \mathrm{ml}^{-1}\right)}\right)$ and were spotted at $\sim 0 \cdot 5 \mu \mathrm{l}$ per spot with a replica device (Oxoid) onto plates containing different drugs, along with 10 -fold serial dilutions. Fluconazole and miconazole, in $0 \cdot 1 \%$ DMSO, were prepared to final concentrations of 5 and $0 \cdot 3 \mu \mathrm{g} \mathrm{ml}^{-1}$, respectively. Cells were grown on SD media containing $0 \cdot 1 \%$ DMSO in the absence or presence of drugs for 2 days at $30^{\circ} \mathrm{C}$.

\section{RESULTS AND DISCUSSION}

\section{ScNdt80p failed to activate CDR1p-lacZ in S. cerevisiae}

CaNdt80p was annotated as the C. albicans homologue of the $S$. cerevisiae transcription factor $\mathrm{ScNdt80p}$ due to the high degree of identity between the $\mathrm{C}$ terminal of CaNdt80p and the $\mathrm{N}$ terminal of ScNdt80p, a known DNA-binding domain (Fig. 1). Interestingly, there is no similarity between the $\mathrm{N}$ terminal of CaNdt80p and the $\mathrm{C}$ terminal of ScNdt80p (Chen et al., 2004). Thus, to investigate the closeness of functionality between these two proteins, we wished to determine whether ScNdt80p, like CaNdt80p, can activate CDR1p-lacZ in $S$. cerevisiae. The $\beta$-Gal activity of CDR $1 p-l a c Z$ was determined by liquid assay, and the results are summarized in Fig. 2 . CaNDT80 in a high-copy-number vector (pRS426) increased the expression of CDR1p-lacZ approximately fivefold in $S$. cerevisiae SLO5, consistent with our previous report (Chen et al., 2004). On the other hand, ScNDT80 in the same vector did not affect the expression of CDR1p-lac $Z$ in $S$. cerevisiae SLO71. Since ScNDT80 is tightly regulated and expressed only during meiosis (Pak \& Segall, 2002), we constructed a chimera in which ScNDT80 was under the control of the CaNDT80 promoter (LOB116) to further determine the effect of ScNdt80p in activating the expression of CDR1placZ. The expression of CDR1p-lacZ was not induced by ScNdt80p in S. cerevisiae SLO79 (Fig. 2), even though the $S c N D T 80$ gene under the control of the CaNDT80 promoter was expressed (Fig. 3a, lane 12). The fact that $\mathrm{ScNdt} 80 \mathrm{p}$ failed to activate $C D R 1 p-l a c Z$ may be due to the difference in activation domain between ScNdt80p and CaNdt80p.

\section{The DNA-binding domain of ScNdt80p can functionally complement that of CaNdt80p in S. cerevisiae}

Since there is high degree identity between the $\mathrm{C}$ terminal of CaNdt80p and the DNA-binding domain of ScNdt80p, it is 
(a)

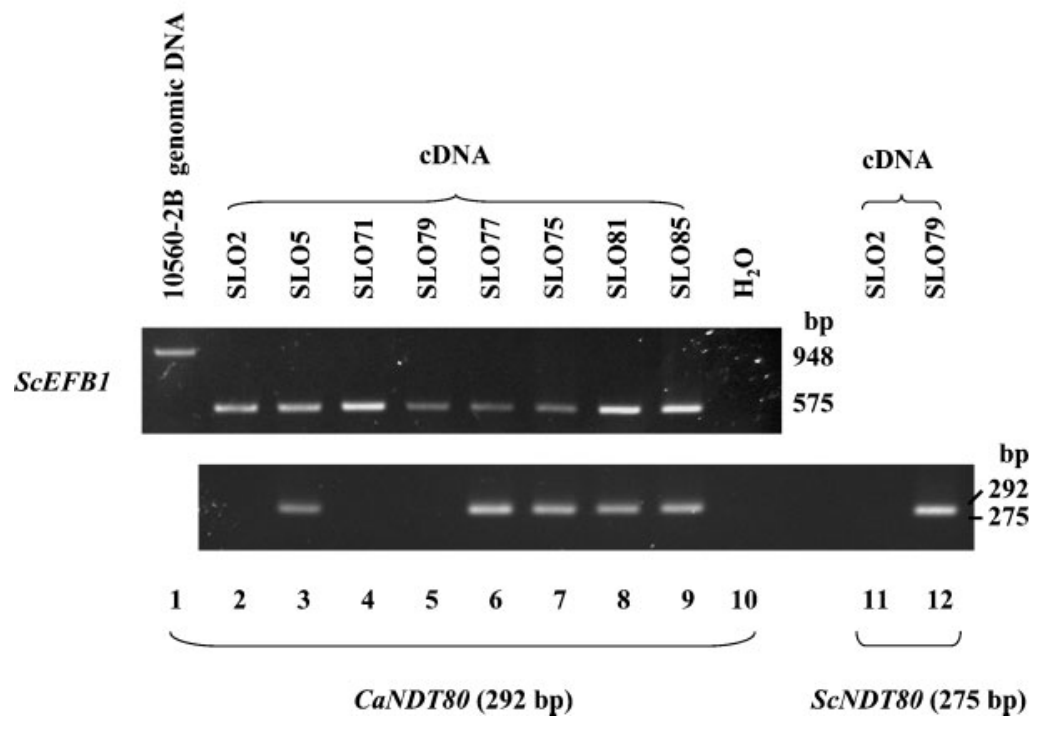

(b)

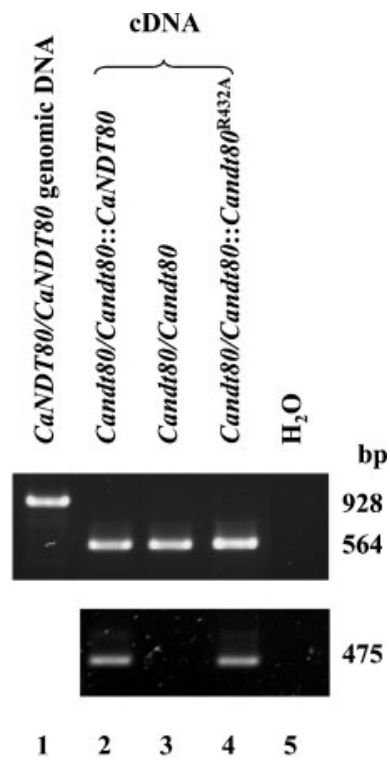

Fig. 3. Expression of different chimeras in S. cerevisiae and both CaNDT8O and Candt80 R432A alleles in C. albicans. (a) RTPCR was performed with primers specific for ScEFB1, ScNDT80 and CaNDT80. Total RNA was isolated from different S. cerevisiae strains. The template for lane 1 was genomic DNA from the parental S. cerevisiae strain (10560-2B). The remaining templates were cDNAs from different $S$. cerevisiae strains: lane 2, SLO2; lane 3, SLO5; lane 4, SLO71; lane 5, SLO79; lane 6, SLO77; lane 7, SLO75; lane 8, SLO81; lane 9, SLO85; lane 10, water as a negative control; lane 11, SLO5; and lane 12, SLO79. Lanes 2-10 were from reactions containing primers for amplifying CaNDT80, and lanes 11-12 were for ScNDT80. (b) RT-PCR was performed with primers specific for CaEFB1 and CaNDT80, separately. The total RNAs were isolated from different $C$. albicans strains. The template for lane 1 was genomic DNA from the wild-type C. albicans strain (SC5314), and the remaining templates were cDNAs from different strains: lane 2, Candt80/Candt80:: CaNDT80 (YLO137); lane 3, Candt80/Candt80 (YLO133); lane 4, Candt80/Candt80: : Candt80 R432A (YLO263); lane 5, water as negative control. The sizes of the RT-PCR products are shown on the right.

possible that the DNA-binding domain of ScNdt80p can functionally complement the C-terminal putative DNAbinding domain of CaNdt80p. To assess this possibility, we constructed a chimera containing the promoter and the $\mathrm{N}$ terminal of CaNdt80p fused with the DNA-binding domain of ScNdt80p (LOB114). The results of the $\beta$-Gal liquid assay are summarized in Fig. 2. This chimera in SLO75 induced the expression of CDR1p-lacZ approximately threefold. Consistently, the chimera containing the promoter and the $\mathrm{N}$ terminal of CaNdt80p fused with the full-length ORF of ScNdt80p in SLO81 also induced the expression of CDR1plac $Z$ approximately threefold. In contrast, overexpression of the $\mathrm{N}$ terminal of CaNdt80p alone in SLO77 did not have any effect on the expression of CDR1p-lacZ. These data suggest that CaNdt80p lacking the putative DNA-binding domain failed to activate $C D R 1 p-l a c Z$ in $S$. cerevisiae. Furthermore, the DNA-binding domain of ScNdt80p could complement the function possessed by the putative DNAbinding domain of CaNdt80p. Therefore, this putative domain shares at least one function with the DNA-binding domain of ScNdt80p.

\section{CaNdt80p with a mutation of the DNA-binding domain failed to activate CDR1p-lacZ in S. cerevisiae}

The DNA-binding capability of ScNdt80p decreased approximately 19-, 27- and 14-fold when the DNA-binding domain contained a mutation at residues Arg111, Arg177 and Arg254, respectively. Several homologues of ScNdt80p from higher eukaryotes, including proteins from Neurospora crassa, Dictyostelium discoideum, Caenorhabditis elegans, Drosophila melanogaster and humans, have been reported (Montano et al., 2002). Interestingly, Arg177 and Arg254 are absolutely conserved, and Arg111 is highly conserved (Arg or Lys), in all these proteins (Montano et al., 2002). Since the DNA-binding domain of CaNdt80p is important for activating CDR1p-lacZ in S. cerevisiae, CaNdt80p with mutations of the DNA-binding domain could fail to activate CDR1p-lacZ. To assess this possibility, we mutated the $\operatorname{Arg} 432$ amino acid of CaNdt80p, corresponding to ScNdt80p residue Arg177, known to be the most important residue for the DNA-binding activity of ScNdt80p (Fig. 1b) 
(Montano et al., 2002). We found that overexpression of Cand $t 80^{\mathrm{R} 432 \mathrm{~A}}$ in the SLO85 strain failed to activate CDR1placZ in S. cerevisiae (Fig. 2). In addition, this is also consistent with the concept that this domain of CaNdt80p is important for the activity associated with DNA binding.

\section{Construction of the Candt80/ Candt80 : : Candt80 ${ }^{\mathrm{R432A}}$ C. albicans strain}

Though we showed that the DNA-binding domain of CaNdt80p is important for activating CDR1p-lacZ in $S$. cerevisiae, we were still not sure if the DNA-binding domain of CaNdt80p indeed plays a role in drug resistance in $C$. albicans. To test this hypothesis, we reintroduced the Candt $80^{\mathrm{R} 432 \mathrm{~A}}$ mutated allele into the locus of CaNDT80 in the Candt80/Candt80 mutant to determine if this mutated allele, like the wild-type CaNDT80 gene, could restore the drug susceptibility phenotype to the Candt80/Candt80 mutant. After assessing the constructed genome with PCR and sequencing, the expression of this mutated allele in the reintroduced Candt80/Candt80::Candt $80^{\mathrm{R} 432 \mathrm{~A}}$ strain was also determined by RT-PCR (Fig. 3b), along with the Candt80/Candt80:: CaNDT80 strain. First, there was no genomic DNA contamination in the preparations of total RNA, since none of the 928 bp intron-containing CaEFB1
DNA fragment was amplified from cDNA samples (Fig. 3b, lanes 2-4). The wild-type CaNDT80 (Fig. 3b, lane 2) and Candt80 ${ }^{\mathrm{R} 432 \mathrm{~A}}$ (Fig. 3b, lane 4) alleles were expressed. We also determined the level of both the CaNDT80 and the Cand $80^{\mathrm{R} 432 \mathrm{~A}}$ alleles by real-time $\mathrm{PCR}$, and the results indicated that both alleles were expressed at the same level.

\section{The Candt $80^{\mathrm{R432A}}$ allele with a mutation of the DNA-binding domain failed to complement Candt80/Candt80 mutant cells}

Consequently, to further elucidate the function of the DNAbinding domain of CaNdt80p in C. albicans, we analysed the drug susceptibility of different strains by the Etest assay and by the agar dilution method. The Etest results are shown in Fig. 4(a). The MIC to fluconazole of the Candt80/Candt80 cells was $0.5 \mathrm{mg} \mathrm{l}^{-1}$. The MICs to fluconazole of the Candt80/Candt80 mutant cells containing a copy of the wild-type CaNDT80 gene (Candt80/Candt80::CaNDT80) increased to $1.5 \mathrm{mg} \mathrm{l}^{-1}$. Thus, the wild-type CaNDT80 gene conferred drug resistance on the Candt80/Candt80 mutant cells. In contrast, the Candt80/Candt80: : Candt80 ${ }^{\mathrm{R} 432 \mathrm{~A}}$ cells were as sensitive to the drug as the Candt80/Candt80 mutant cells. The results of the agar dilution method are shown in Fig. 4(b). All cells tested grew in the absence of drug. The

(a)
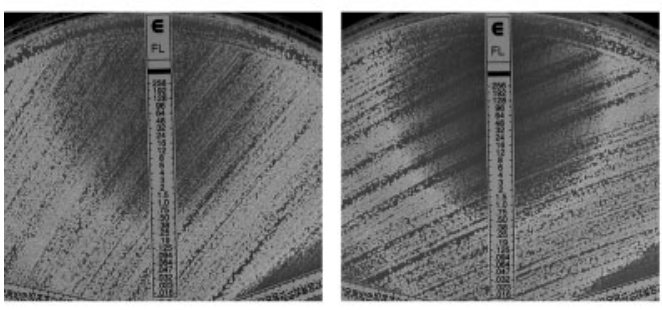

CsNDT80/CaNDT80

\section{Candt80/Candt80:: CaNDT80}
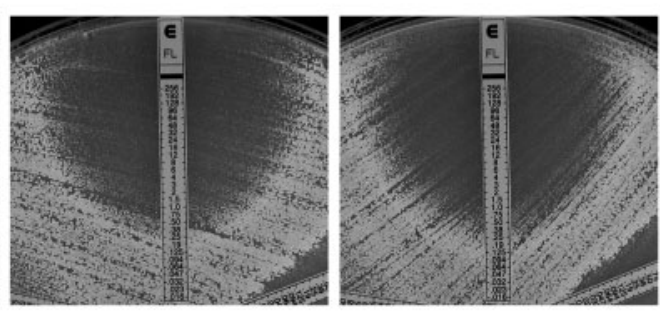

\section{Candt80/Candt80}

\section{Candt80/Candt80:: Candt $80^{\mathrm{R} 432 \mathrm{~A}}$}

(b)

CaNDT80/CaNDT80

Candt80/Candt80::CaNDT80

Candt80/Candt80

Candt80/Candt80::Candt80 ${ }^{\mathrm{R} 432 \mathrm{~A}}$
Control

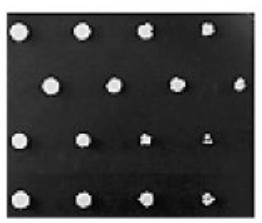

$\begin{array}{llll}10^{4} & 10^{3} & 10^{2} & 10^{1}\end{array}$
$5 \mu \mathrm{g}$ fluconazole $0.3 \mu \mathrm{g}$ miconazole $\mathrm{ml}^{-1}$

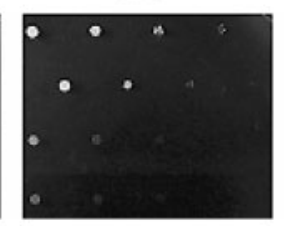

$\mathrm{ml}^{-1}$

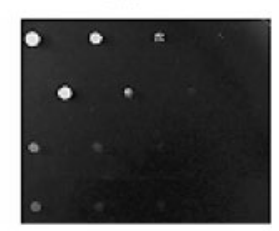

Cell number

Fig. 4. The Candt $80^{\mathrm{R} 432 \mathrm{~A}}$ allele failed to complement Candt80/Candt 80 mutant cells. Susceptibility to azole drugs was

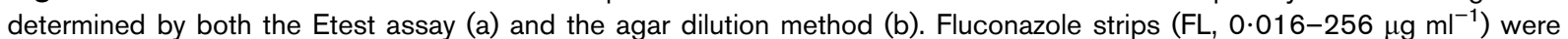
used for the Etest. For agar dilution, cells were grown on media in the absence of drug, or in the presence of $5 \mu \mathrm{g}$ fluconazole $\mathrm{ml}^{-1}$ or $0.3 \mu \mathrm{g} \mathrm{miconazole} \mathrm{ml}^{-1}$. The results of both assays for four tested strains, CaNDT80/CaNDT80 (SC5314), Candt80/ Candt80:: CaNDT80 (YLO137), Candt80/Candt80 (YLO133), and Candt80/Candt80::Candt80 ${ }^{\mathrm{R} 432 \mathrm{~A}}$ (YLO263) were photographed after 2 days growth at $30^{\circ} \mathrm{C}$. 
Candt80/Candt80 mutant cells with the wild-type CaNDT80 allele were more susceptible to the drug than the wild-type cells. This may be due to the dosage effect described previously (Chen et al., 2004; Kohler \& Fink, 1996). The Candt80/Candt80::CaNDT80 cells with the wild-type CaNDT80 allele were more resistant to drugs than the Candt80/Candt80 mutant cells, consistent with the Etest results and our previous report that CaNdt80p is involved in drug resistance (Chen et al., 2004). It was noteworthy that the Candt80/Candt80:: Candt $80^{\mathrm{R} 432 \mathrm{~A}}$ cells with the mutated Candt $80^{\mathrm{R} 432 \mathrm{~A}}$ allele were as sensitive to drugs as the Candt80/Candt80 mutant cells. The results of both the Etest assay and the agar dilution method suggested that, unlike the wild-type CaNDT80 allele, Candt $80^{\mathrm{R} 432 \mathrm{~A}}$ failed to complement the lack of drug resistance of the Candt80/ Candt 80 cells.

We have shown that the fact that $\mathrm{ScNdt} 80 \mathrm{p}$ failed to activate CDR1p-lacZ in S. cerevisiae may have been due to the differences in the activation domains between ScNdt80p and CaNdt80p. Nevertheless, we have been able to show that the DNA-binding domain of ScNdt80p could complement that of CaNdt80p to activate CDR1p-lacZ. Furthermore, CaNdt80p containing a mutation of the DNA-binding domain failed to complement the drug sensitivity caused by a null mutation in C. albicans. Thus, the DNA-binding domain of CaNdt80p is critical for the drug resistance of $C$. albicans, and it may have the same DNA-binding site as that of ScNdt80p.

\section{ACKNOWLEDGEMENTS}

This work was in part supported by grants 94-2320-B-400-001 and 942320-B-009-001 from the Taiwan National Science Council, and grant CL-094-PP-05 from the Taiwan National Health Research Institutes.

\section{REFERENCES}

Abi-Said, D., Anaissie, E., Uzun, O., Raad, I., Pinzcowski, H. \& Vartivarian, S. (1997). The epidemiology of hematogenous candidiasis caused by different Candida species. Clin Infect Dis 24, 1122-1128.

Beck-Sague, C. \& Jarvis, W. R. (1993). Secular trends in the epidemiology of nosocomial fungal infections in the United States, 1980-1990. National Nosocomial Infections Surveillance System. J Infect Dis 167, 1247-1251.

Chen, Y. C., Chang, S. C., Sun, C. C., Yang, L. S., Hsieh, W. C. \& Luh, K. T. (1997). Secular trends in the epidemiology of nosocomial fungal infections at a teaching hospital in Taiwan, 1981 to 1993. Infect Control Hosp Epidemiol 18, 369-375.

Chen, C. G., Yang, Y. L., Shih, H. I., Su, C. L. \& Lo, H. J. (2004). CaNdt80 is involved in drug resistance in Candida albicans by regulating CDR1. Antimicrob Agents Chemother 48, 4505-4512.

Christianson, T. W., Sikorski, R. S., Dante, M., Shero, J. H. \& Hieter, P. (1992). Multifunctional yeast high-copy-number shuttle vectors. Gene $110,119-122$.

Chu, S. \& Herskowitz, I. (1998). Gametogenesis in yeast is regulated by a transcriptional cascade dependent on Ndt80. Mol Cell 1, 685-696.
Chu, S., DeRisi, J., Eisen, M., Mulholland, J., Botstein, D., Brown, P. O. \& Herskowitz, I. (1998). The transcriptional program of sporulation in budding yeast. Science 282, 699-705.

Coste, A. T., Karababa, M., Ischer, F., Bille, J. \& Sanglard, D. (2004). $T A C 1$, transcriptional activator of $C D R$ genes, is a new transcription factor involved in the regulation of Candida albicans $\mathrm{ABC}$ transporters CDR1 and CDR2. Eukaryot Cell 3, 1639-1652.

Edwards, E. J. J. (1990). Candida species. In Principles and Practice of Infectious Diseases, pp. 1943-1958. Edited by G. L. Mandell, R. G. Douglas \& J. E. Bennett. London: Churchill-Livingstone.

Gillum, A. M., Tsay, E. Y. \& Kirsch, D. R. (1984). Isolation of the Candida albicans gene for orotidine- $5^{\prime}$-phosphate decarboxylase by complementation of S. cerevisiae ura3 and E. coli pyrF mutations. Mol Gen Genet 198, 179-182.

Hung, C. C., Chen, Y. C., Chang, S. C., Luh, K. T. \& Hsieh, W. C. (1996). Nosocomial candidemia in a university hospital in Taiwan. J Formos Med Assoc 95, 19-28.

Karababa, M., Coste, A. T., Rognon, B., Bille, J. \& Sanglard, D. (2004). Comparison of gene expression profiles of Candida albicans azole-resistant clinical isolates and laboratory strains exposed to drugs inducing multidrug transporters. Antimicrob Agents Chemother 48, 3064-3079.

Kofron, M., Demel, T., Xanthos, J. \& 7 other authors (1999). Mesoderm induction in Xenopus is a zygotic event regulated by maternal VegT via TGFbeta growth factors. Development 126, 5759-5770.

Kohler, J. R. \& Fink, G. R. (1996). Candida albicans strains heterozygous and homozygous for mutations in mitogen-activated protein kinase signaling components have defects in hyphal development. Proc Natl Acad Sci U S A 93, 13223-13228.

Lopez-Ribot, J. L., McAtee, R. K., Lee, L. N., Kirkpatrick, W. R., White, T. C., Sanglard, D. \& Patterson, T. F. (1998). Distinct patterns of gene expression associated with development of fluconazole resistance in serial Candida albicans isolates from human immunodeficiency virus-infected patients with oropharyngeal candidiasis. Antimicrob Agents Chemother 42, 2932-2937.

Marger, M. D. \& Saier, M. H., Jr (1993). A major superfamily of transmembrane facilitators that catalyse uniport, symport and antiport. Trends Biochem Sci 18, 13-20.

Michaelis, S. \& Berkower, C. (1995). Sequence comparison of yeast ATP-binding cassette proteins. Cold Spring Harb Symp Quant Biol 60, 291-307.

Montano, S. P., Cote, M. L., Fingerman, I., Pierce, M., Vershon, A. K. \& Georgiadis, M. M. (2002). Crystal structure of the DNA-binding domain from Ndt80, a transcriptional activator required for meiosis in yeast. Proc Natl Acad Sci U S A 99, 14041-14046.

Pak, J. \& Segall, J. (2002). Regulation of the premiddle and middle phases of expression of the NDT80 gene during sporulation of Saccharomyces cerevisiae. Mol Cell Biol 22, 6417-6429.

Pfaller, M. A., Jones, R. N., Messer, S. A., Edmond, M. B. \& Wenzel, R. P. (1998). National surveillance of nosocomial blood stream infection due to species of Candida other than Candida albicans: frequency of occurrence and antifungal susceptibility in the SCOPE Program. Diagn Microbiol Infect Dis 30, 121-129.

Pfaller, M. A., Jones, R. N., Doern, G. V., Sader, H. S., Messer, S. A., Houston, A., Coffman, S. \& Hollis, R. J. (2000). Bloodstream infections due to Candida species: SENTRY antimicrobial surveillance program in North America and Latin America, 1997-1998. Antimicrob Agents Chemother 44, 747-751.

Pfaller, M. A., Diekema, D. J., Messer, S. A., Boyken, L. \& Hollis, R. J. (2003). Activities of fluconazole and voriconazole against 1,586 
recent clinical isolates of Candida species determined by broth microdilution, disk diffusion, and Etest methods: report from the ARTEMIS Global Antifungal Susceptibility Program, 2001. J Clin Microbiol 41, 1440-1446.

Sanglard, D., Ischer, F., Monod, M. \& Bille, J. (1996). Susceptibilities of Candida albicans multidrug transporter mutants to various antifungal agents and other metabolic inhibitors. Antimicrob Agents Chemother 40, 2300-2305.

Schaller, M., Bein, M., Korting, H. C., Baur, S., Hamm, G., Monod, M., Beinhauer, S. \& Hube, B. (2003). The secreted aspartyl proteinases Sap1 and Sap2 cause tissue damage in an in vitro model of vaginal candidiasis based on reconstituted human vaginal epithelium. Infect Immun 71, 3227-3234.
Schmitt, M. E., Brown, T. A. \& Trumpower, B. L. (1990). A rapid and simple method for preparation of RNA from Saccharomyces cerevisiae. Nucleic Acids Res 18, 3091-3092.

Sherman, F. (2002). Getting started with yeast. Methods Enzymol 350, 3-41.

Tapia, C., Leon, E. \& Palavecino, E. (2003). Antifungal susceptibility of yeasts by Etest. Comparison of 3 media. Rev Med Chil 131, 299302 (in Spanish).

Vanden Bossche, H., Warnock, D. W., Dupont, B. \& 7 other authors (1994). Mechanisms and clinical impact of antifungal drug resistance. J Med Vet Mycol 32, 189-202.

Yang, Y. L. \& Lo, H. J. (2001). Mechanisms of antifungal agent resistance. J Microbiol Immunol Infect 34, 79-86. 